\title{
Hyperprogressive Disease In Cervical Small Cell Carcinoma Treated By Immune Checkpoint Inhibitor
}

This article was published in the following Dove Press journal: OncoTargets and Therapy

\section{Zihan Xu* \\ Liying Chen* \\ Linpeng Zheng \\ Qiao Yang \\ Mingjing Chen \\ Jianmin Wang \\ Guangkuo Zhu \\ Zhengtang Chen \\ Jianguo Sun}

Cancer Institute of People's Liberation Army, Xinqiao Hospital, Army Medical University, Chongqing, People's Republic of China

*These authors contributed equally to this work
Correspondence: Jianguo Sun

Cancer Institute of People's Liberation Army, Xinqiao Hospital, Army Medical University, Chongqing, People's Republic of China

Tel +862368774490

Fax +862368774631

Email sunjg09@aliyun.com
Abstract: A new progression pattern, hyperprogressive disease (HPD), has been recently acknowledged in cancer patients accepted immune checkpoint inhibitors (ICIs). We report a unique case of cervical small cell carcinoma which showed primary resistance to pembrolizumab and was with a rapid radiological progression after the initiate of ICIs treatment. However, the detection results of multiple predictive biomarkers suggested that the patient was eligible for ICIs treatment. The whole exome sequencing showed that AKT1 E17K mutation was high $(26.316 \%)$ in tumor tissue, and dynamic monitoring of circulating tumor DNA indicated that AKT1 E17K mutation rate was increasing successively and highly consistent with tumor growth in peripheral blood. Therefore, the correlation between AKT1 E17K mutation and HPD, and the role of AKT1 E17K mutation in identifying patients who might not benefit from ICIs treatment need to be further studied.

Keywords: hyperprogressive disease, cervical small cell carcinoma, immune checkpoint inhibitor

\section{Introduction}

Cervical cancer represents the fourth most common malignancy affecting women around the world. ${ }^{1}$ Despite the improvements in screening and treatment of earlystage disease, a proportion of patients will be diagnosed with persistent, advanced stage, or recurrent cervical cancer. For this subgroup of patients, including small cell carcinoma subtype, systemic chemotherapy is still the base of treatment. ${ }^{2,3}$ Besides, as for vaginal recurrences, radiotherapy and/or pelvic exenteration represent the most frequently adopted treatment, but unfortunately, the prognosis remains poor. ${ }^{4,5}$

Immune checkpoint inhibitors (ICIs), especially programmed cell death-1/ programmed death-ligand 1 (PD-1/PD-L1), have exploited a promising field for cancer treatment. ${ }^{6,7}$ However, a small part of patients seems to not benefit from ICIs and, on the contrary, these treatments are likely to accelerate tumor growth. In fact, Champiat et al reported a new progression pattern, hyperprogressive disease (HPD), in patients treated with ICIs. These patients experienced disease progression by RECIST criteria with a $\geq 2$-fold increase in the tumor growth rate (TGR) between the experimental periods (from baseline to the first tumor evaluation) and the reference period (prior to treatment onset). ${ }^{8}$ At present, there was no idea about the predictors for HPD. Biomarkers for outcomes during ICIs treatment are also not available, therefore, developing predictive biomarkers for ICIs is greatly necessary. ${ }^{9}$ 


\section{Case Report And Genetic Analysis}

An otherwise healthy 49-year-old woman with irregular vaginal bleeding was referred to Department of Gynecology and Obstetrics on May 27, 2016. The schematic course of diagnosis and treatment of the patient was shown in Figure 1A. Cervical biopsy suggested cervical malignancy with poor differentiation. The patient underwent extensive excision of cervical tumor on July 21, 2016. Postoperative

A

$\begin{array}{ccccc}\text { 2016.5.27 } & \text { 2016.7.21 } & \text { 2016.8.17 } & \text { 2016.11.23 } & \text { 2017.4.6 } \\ \text { Diagnosis } & \text { Surgery } & \text { Chemotherapy } & \text { PD-1 therapy started } & \text { Sleep apnea-hypopnea syndrome }>>> \\ \text { Cervical biopsy } & \begin{array}{c}\text { Extensive resection of } \\ \text { small cell cervical } \\ \text { carcinoma: }\end{array} & \text { Adjuvant chemotherapy } & \text { Six cycles of Pembrolizumab } & \begin{array}{c}\text { Occur repeatedly and } \\ \text { aggravate gradually }\end{array}\end{array}$

Panhysterectomy, Bilateral

salpingectomy, cleaning

operation of pelvic lymph

nodes, and resection of vaginal wall nodule

B
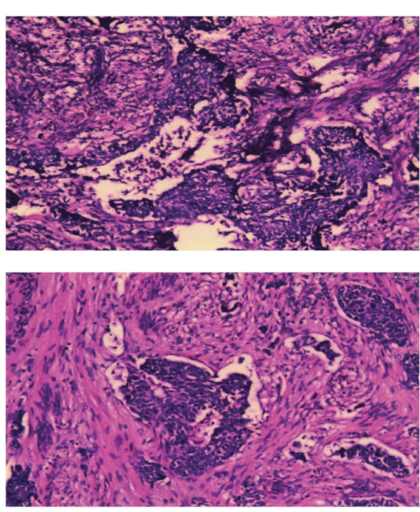

C
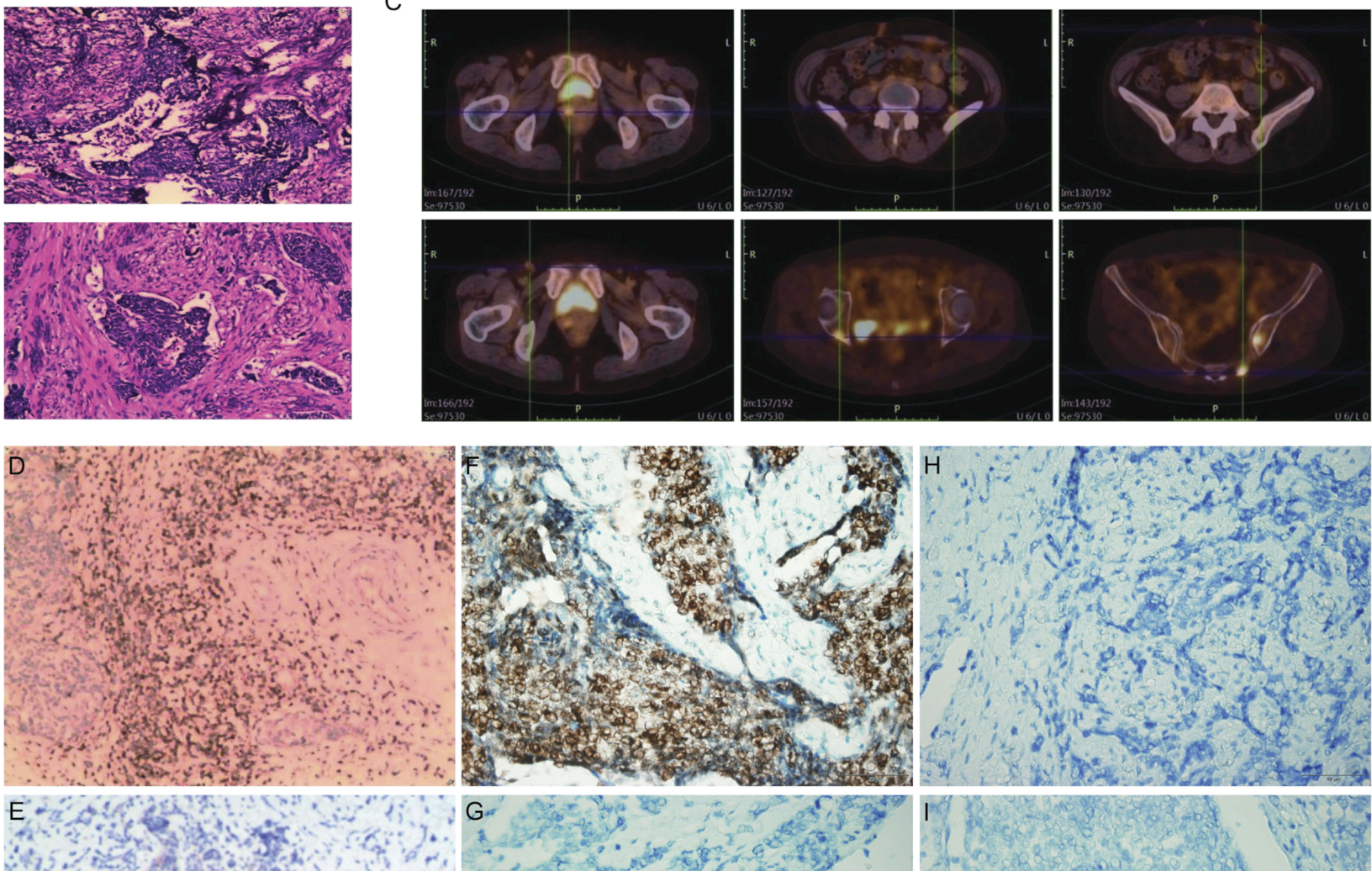

1) 13
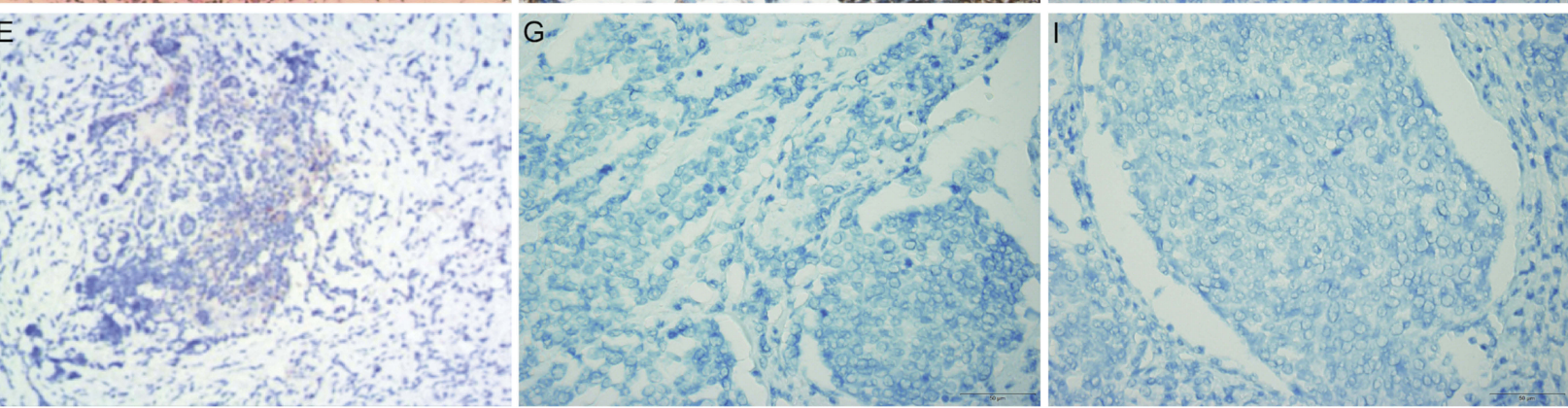

Figure I Histologic and radiographic findings, and immunohistochemical staining for CD3+ TILs, PD-LI, and MLHI, MSH2, MSH6 and PMS2 expression in cervical small cell carcinoma tissue before PD-I inhibitor treatment. (A) Clinical course of the patient. (B) Immunohistochemical staining of excisional tumor tissue demonstrated small cell cervical carcinoma, infiltrating the full-thickness cervix. (C) FDG-PET/CT overview showed multiple lesions of increased FDG metabolism in the field of operation, left lateral psoas muscle, right groin district lymph nodes, and multiple bones throughout the body. (D) Abundant CD3+ TILs infiltrated in cervical small cell carcinoma tissue. (E) Missing immunohistochemical staining for PD-LI in tumor cells. (F) Representative immunohistochemical staining for MLHI in tumor cells, and missing immunohistochemical staining of MSH2 (G), MSH6 (H), and PMS2 (I) in tumor cells. Original magnification $\times 400$.

Abbreviations: PD-I, programmed death-receptor I; TILs, tumor-infiltrating lymphocytes; PD-LI, programmed death ligand I; FDG-PET/CT, I8F-fluorodeoxyglucose (FDG)-positron emission tomography (PET)/computed tomography (CT). 
pathological examination demonstrated small cell carcinoma infiltrating the full thickness of the cervix and involving the full layer of vaginal fornix (Figure 1B). On September 14, 2016, radiological examination with positron emission tomography-computed tomography (PET-CT) scan indicated multiple lesions of increased FDG metabolism, including the field of operation (residual tumor), the sites of multiple bones, the left lateral psoas muscle and the right groin district (bone and lymph node metastases) (Figure 1C).

The patient received adjuvant chemotherapy with one cycle of etoposide combined with nedaplatin and two cycles of paclitaxel combined with cisplatin after surgery according to the standard. However, after chemotherapy, serious adverse events have appeared, including nausea, vomiting, fatigue, abdominal pain, and III-IV ${ }^{\circ}$ myelosuppression. Meanwhile, magnetic resonance imaging (MRI) showed multiple tiny bony metastases. As the patient could not tolerate the druginduced toxicity and the efficacy was evaluated as progressive disease (PD), ICIs was then considered. Analysis of biomarkers for ICIs efficacy prediction showed that the patient had dMMR tumors with the loss of MSH2, MSH6, and PMS2 expression. Tumor-infiltrating lymphocytes were abundant. Although PD-L1 IHC (SP 142, Roche) in tumor tissue was negative $(<1 \%$ ), but PD-L1 mRNA in tumor tissue was 33.9\% (Figure 1D-I). For further analysis, tumor tissue and blood of the patient were used for extracting DNA by Maxwell ${ }^{\circledR}$ RSC Instrument according to the manufacturer's instruction of the Maxwell RSC Tissue DNA Kit and Maxwell RSC Blood DNA Kit (Promega, Madison, WI, USA) for whole-exome sequencing (WES). Based on an analysis of reads that uniquely aligned to the reference genome and for which the potential PCR duplicates were removed, an average coverage of $75.24 \mathrm{X}$ and $118.8 \mathrm{X}$ was achieved with $91.76 \%$ and $87.89 \%$ of the targeted bases being covered at $10 \mathrm{X}$ or greater read depth for normal tissue and tumor, respectively. WES of tumor tissue found 1257 tumor gene mutations, $53.5 \%$ of the chromosome copy number abnormalities, and $0.58 \%$ microsatellite instability, suggesting that the patient was eligible for and could benefit from ICIs treatment.

Therefore, the patient received six cycles of pembrolizumab, a PD-1 inhibitor, 150mg q3w, from November 2016 to March 2017. Meanwhile, monitoring of circulating tumor DNA (ctDNA) mutation abundance, detection of circulating tumor cells (CTCs) amount and tumor markers, and imaging evaluation were performed every two cycles. Throughout the course of treatment, no obvious drug-related adverse events were observed except for mild hyperthyroidism, which was controlled after methimazole treatment. Monitoring of the efficacy during the course of treatment showed that the mutation abundance of ctDNA in peripheral blood and the amount of CTCs had successively increased, and the tumor volume had rapidly increased. MRI showed that the increase of pelvic lesions was greater than $50 \%$ in the first efficacy evaluation (two months after ICIs treatment compared to pre-treatment), and new metastases appeared. Chest computer tomography (CT) revealed pulmonary nodules, indicating tumor pulmonary metastases (Figure 2).

The WES and ctDNA mutation analysis showed that AKT1 E17K mutation was high $(26.316 \%)$ in tumor tissue, and the mutation rate increasing successively and highly consistent with tumor growth in peripheral blood (Table 1).

\section{Discussion}

The clinical application of ICIs has exploited an encouraging era of anticancer therapy. ${ }^{10}$ But, a new pattern of progression, HPD which was observed in $9 \%$ of patients treated with anti-PD-1/PD-L1, has drawn extensive attention. ${ }^{8}$ It is related to worse overall survival.

This case showed dramatic hyperprogressive disease after the initiate of pembrolizumab according to Kato's criteria, as time-to-treatment failure (TTF) $<2$ months, $>50 \%$ increase in tumor burden compared to pre-immunotherapy imaging, and $>2$-fold increase in TGR. ${ }^{11}$ Recently HPD was observed in some patients who accepted ICIs treatment, which is relative to older age (> 65 years old) and slower-growing tumors at baseline. ${ }^{8}$ Besides, some activation mutations may also be relative to the occurrence of HPD. Accelerated tumor growth could be associated with the blockade of PD-1/PD-L1 signaling pathway in combination with genetic mutations such as MDM2 family amplification. Kato et al reported, among 155 patients treated with anti-PD1/PDL1 monotherapy, all six individuals with TTF $<2$ months existed MDM2/ MDM4 amplification. ${ }^{11}$ However, the mechanism of HPD in this patient is unclear. It may be related to the gene mutation leading to the activation of the corresponding signaling pathway. The WES and ctDNA mutation analysis indicated that the mutation rate of AKT1 E17K was high in tumor tissue and the mutation frequency of ctDNA increased successively and was highly consistent with tumor growth in peripheral blood, suggesting that AKT1 E17K activation mutation is likely to be an important molecular mechanism underlying HPD.

As for adverse events, it's only occurred mild hyperthyroidism which was controlled after methimazole treatment. 
A
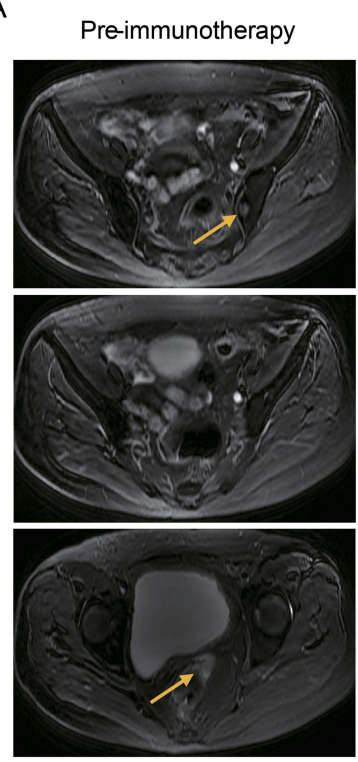

Post-immunotherapy
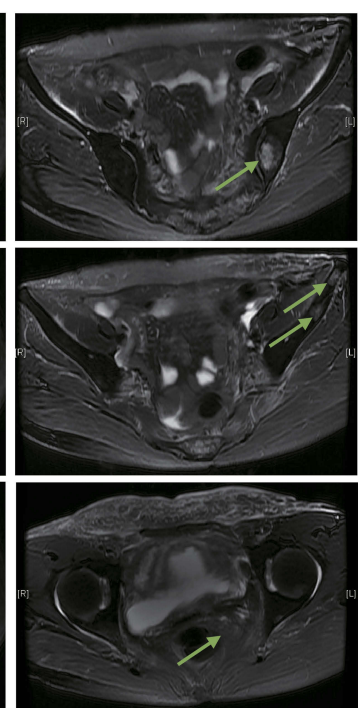

B

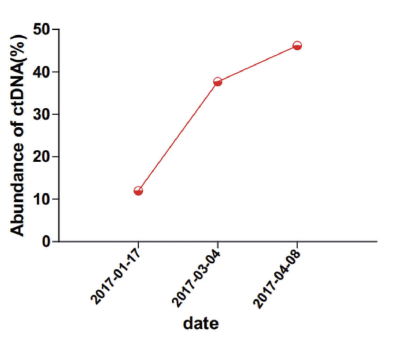

CA125

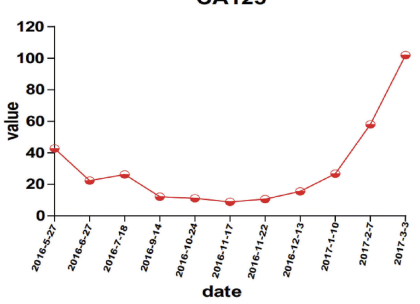

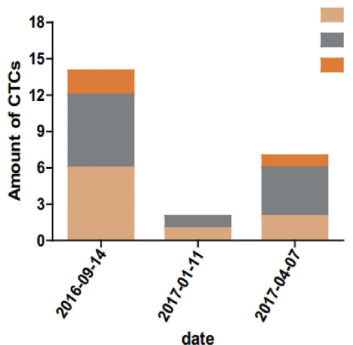

CEA

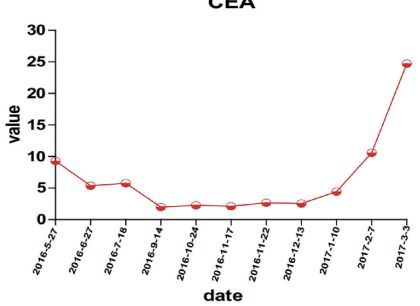

Figure 2 Radiographic changes pre- and post-immunotherapy and variation tendency of immunity indexes and tumor markers during immunotherapy. (A) MRI of pelvic cavity pre- and post-immunotherapy: The target tumor volume increased, and new metastases appeared. Arrows indicate the tumor lesions pre- (yellow arrows) and postimmunotherapy (green arrows). (B) The mutation abundance of ctDNA in peripheral blood and the amount of CTCs increased successively. (C) Variation tendency of tumor markers was observed during process of diagnosis and treatment: tumor markers gradually decreased after surgery, and then returned to normal; after the start of immunotherapy, tumor markers continued to rise above the normal.

Abbreviations: ctDNA, circulating tumor DNA; CTCs, circulating tumor cells.

Table I The WES And ctDNA Mutation Analysis During Immunotherapy

\begin{tabular}{|l|l|l|l|l|}
\hline $\begin{array}{l}\text { Detection } \\
\text { Time }\end{array}$ & Gene & $\begin{array}{l}\text { Mutation } \\
\text { Site }\end{array}$ & $\begin{array}{l}\text { Mutation } \\
\text { Frequency } \\
\text { In Tumor } \\
\text { Tissue }\end{array}$ & $\begin{array}{l}\text { Mutation } \\
\text { Frequency } \\
\text { Of ctDNA } \\
\text { Detection }\end{array}$ \\
\hline 20I7.I.I7 & AKTI & p.EI7K & $26.316 \%$ & $11.344 \%$ \\
& PIK3CA & p.E545K & $5.000 \%$ & $0.141 \%$ \\
& JakI & Non & 0 & 0 \\
& Jak2 & Non & 0 & 0 \\
& B2M & Non & 0 & 0 \\
\hline 2017.3 .4 & AKTI & p.EI7K & $26.316 \%$ & $29.982 \%$ \\
& PIK3CA & p.E545K & $5.000 \%$ & $0.031 \%$ \\
& JakI & Non & 0 & 0 \\
& Jak2 & Non & 0 & 0 \\
& B2M & Non & 0 & 0 \\
\hline 2017.4 .8 & AKTI & p.EI7K & $26.316 \%$ & $42.340 \%$ \\
& PIK3CA & p.E545K & $5.000 \%$ & $0.102 \%$ \\
& JakI & Non & 0 & 0 \\
& Jak2 & Non & 0 & 0 \\
& B2M & Non & 0 & 0 \\
\hline
\end{tabular}

Abbreviations: WES, whole exome sequencing; ctDNA, circulating tumor DNA.

However, sleep apnea-hypopnea syndrome occurred repeatedly after six cycles of ICIs, and eventually led to the patient's death. The patient had a history of snoring, which is the leading cause of sleep apnea-hypopnea syndrome. Meanwhile, pulmonary metastatic lesions also affected respiratory function, aggravated sleep apnea-hypopnea syndrome.

In summary, this is the first description of genomic profiles of ICIs treated cervical small cell carcinoma with HPD. The case occurred synchronously with clinical deterioration and the radiological finding of HPD. We can't confirm the correlation between the AKT1 E17K mutation and the occurrence of HPD. Prospective studies are needed to demonstrate the specific role of AKT1 E17K mutation during ICIs treatment and to verify if the AKT1 E17K mutation may be a predictive factor of ICIs failure and HPD.

\section{Ethics Approval And Consent For Publication}

Because the patient has died, written informed consent for publication of the clinical details and images was obtained from the family of the patient. The patient's identification has been protected and any information which could be used to reveal the identity of the patient has been removed. The case details and images were approved for publication by Xinqiao Hospital, Army Medical University.

\section{Availability Of Data And Materials}

The datasets analyzed during the current study are available from the corresponding authors on reasonable request. 


\section{Acknowledgments}

The authors sincerely thank Juan $\mathrm{Li}$ and Yongxin $\mathrm{Yu}$ for collecting tissue specimens, English teacher Xiewan Chen (Medical English Department, Army Medical University) for the linguistic modification.

\section{Author Contributions}

All authors contributed to conception and design, selection and acquisition of data, or analysis and interpretation of data; participated in drafting and revising the article; gave final approval of the version to be published; and agree to be accountable for all aspects of the work.

\section{Funding}

The present study was supported by the National Natural Science Foundation of China (No. 81602688, 81773245).

\section{Disclosure}

The authors report no conflicts of interest in this work.

\section{References}

1. Bray F, Ferlay J, Soerjomataram I, Siegel RL, Torre LA, Jemal A. Global cancer statistics 2018: GLOBOCAN estimates of incidence and mortality worldwide for 36 cancers in 185 countries. CA Cancer $J$ Clin. 2018;68(6):394-424. doi:10.3322/caac.21492
2. Eskander RN, Tewari KS. Chemotherapy in the treatment of metastatic, persistent, and recurrent cervical cancer. Curr Opin Obstet Gynecol. 2014;26(4):314-321. doi:10.1097/GCO.0000000000000042

3. Monk BJ, Tewari KS. Evidence-based therapy for recurrent cervical cancer. J Clin Oncol. 2014;32(25):2687-2690. doi:10.1200/JCO. 2014.56.8733

4. Kim HJ, Chang JS, Koom WS, Lee KC, Kim GE, Kim YB. Radiotherapy is a safe and effective salvage treatment for recurrent cervical cancer. Gynecol Oncol. 2018;151(2):208-214. doi:10.1016/j. ygyno.2018.08.029

5. Benedetti PP, Manci N, Bellati F, et al. Vaginectomy: a minimally invasive treatment for cervical cancer vaginal recurrence. Int $J$ Gynecol Cancer. 2009;19(9):1625-1631. doi:10.1111/IGC.0b013 e3181a80a0a

6. Chen L, Han X. Anti-PD-1/PD-L1 therapy of human cancer: past, present, and future. J Clin Invest. 2015;125(9):3384-3391. doi:10.1172/JCI80011

7. Sharma P, Allison JP. The future of immune checkpoint therapy. Science. 2015;348(6230):56-61. doi:10.1126/science.aaa8172

8. Champiat S, Dercle L, Ammari S, et al. Hyperprogressive disease is a new pattern of progression in cancer patients treated by anti-PD-1/ PD-L1. Clin Cancer Res. 2017;23(8):1920-1928. doi:10.1158/10780432.CCR-16-1741

9. George S, Miao D, Demetri GD, et al. Loss of PTEN is associated with resistance to anti-PD-1 checkpoint blockade therapy in metastatic uterine leiomyosarcoma. Immunity. 2017;46(2):197-204. doi:10.1016/j.immuni.2017.02.001

10. Gibney GT, Weiner LM, Atkins MB. Predictive biomarkers for checkpoint inhibitor-based immunotherapy. Lancet Oncol. 2016;17 (12):e542-e551. doi:10.1016/S1470-2045(16)30406-5

11. Kato S, Goodman A, Walavalkar V, Barkauskas DA, Sharabi A, Kurzrock R. Hyperprogressors after immunotherapy: analysis of genomic alterations associated with accelerated growth rate. Clin Cancer Res. 2017;23(15):4242-4250. doi:10.1158/1078-0432.CCR16-3133
OncoTargets and Therapy

\section{Publish your work in this journal}

OncoTargets and Therapy is an international, peer-reviewed, open access journal focusing on the pathological basis of all cancers, potential targets for therapy and treatment protocols employed to improve the management of cancer patients. The journal also focuses on the impact of management programs and new therapeutic

\section{Dovepress}

agents and protocols on patient perspectives such as quality of life, adherence and satisfaction. The manuscript management system is completely online and includes a very quick and fair peer-review system, which is all easy to use. Visit http://www.dovepress.com/ testimonials.php to read real quotes from published authors. 